\title{
Image Noise Level Estimation by Neural Networks
}

\author{
Wang Zhiming ${ }^{1, a^{*}}$, Yuan Guobin ${ }^{2, b}$ \\ ${ }^{1}$ University of Science and Technology Beijing . 30 Xueyuan Road, Haidian District, Beijing 100083 \\ P. R.China \\ ${ }^{2}$ University of Science and Technology Beijing . 30 Xueyuan Road, Haidian District, Beijing 100083 \\ P. R.China \\ a wangzhiming@ies.ustb.edu.cn, byuanguobingo@163.com
}

Keywords: Noise Estimation; Singular Value Decomposition; Neural Networks; Gaussian noise; Hybrid noise.

\begin{abstract}
Aiming at the problem of image noise level estimation, this paper proposes an algorithm for noise estimation by singular value decomposition and neural network. The larger (head) parts of the singular values of an image are mainly affected by main structure of the image, and the rest (tail) parts of the singular values are affected by the intensity of noise. With the increase of noise level, corresponding tail parts of singular values are increased. So, singular values should be good characteristics for noise intensity estimation. Firstly, we add different noise with known intensity on a batch of noise free images, and then select a certain number of fixed size image blocks which standard deviation are minimum from these noisy images. Then singular values of these blocks were fed as the input of the neural network, their corresponding noise standard deviation as the output to train neural network. Finally, in the estimation phase, singular values of noise image were used fed into the trained network to predict the unknown noise intensity. The experimental results show that proposed algorithm is quite promising. It can estimates different types of noise with fast speed and high precise, including Gauss white noise and Hybrid noise.
\end{abstract}

\section{Introduction}

During the acquisition, processing and transmission of a digital image, it is often disturbed by the imaging device and the external environment, which inevitably produce noise and make the image quality degradation. However, preprocessing in image segmentation, edge extraction and target recognition algorithms need to remove the noise in the image to ensure the effectiveness of these algorithms. Therefore, in order to get high quality digital image, denoising is a necessary preprocessing step. Now there were many algorithms can achieve good denoising effect, but most of the algorithms assume that the noise intensity is known, so how to accurately estimate the intensity of noise has been a hot research topic in the field of image restoration.

Probability distribution function were used to describe and quantify the noise in theory. However in practical applications we often use some of its digital features, namely the mean variance and correlation function, to describe noise. Because these features can reflect the characteristics of noise in a certain extent. Zero mean additive Gauss white noise (AWGN) model is widely used in the field of image denoising.

The additive noise in the image can be described by Eq. 1, where $g$ is the noisy image, $f$ is the ideal noise free image, and $n$ is the noise not related to the intensity of image signal.

$$
g(i, j)=f(i, j)+n(i, j)
$$

When the probability density function of $n$ is the Gauss function, the noise image $g$ is the image with additive white Gauss noise. Probability density function of gauss random variable $x$ is:

$$
\mathrm{f}(\mathrm{x})=\frac{1}{\sigma \sqrt{2 \pi}} e^{-\frac{(x-\mu)^{2}}{2 \sigma^{2}}}
$$


In Eq. 2, $x$ represents the grey value of image, mu as the mean of $x$, sigma as the standard deviation of $x$. For zero mean gaussian noise $m u=0$, therefore the noise intensity prediction is to estimate the standard deviation.

At present, many common noise estimation method can be divided into three following categories: (1) filter-based methods [1-3]: this kind of method is usually use mean or median filter to filter the image, and then using the difference between the filtered restoration image and noisy image to estimate the noise. (2) block-based methods [3-5]: the basic idea is, the image is divided into several small pieces, and each piece of the standard deviation of calculated separately, a smaller portions of the standard deviation of mean value were calculated as the estimate of the noise of the image intensity. But this method based on image block didn't considering the influence of the image edge details, and it has poor robustness. (3) transform-based methods [6-8]: this kind of method is using the characteristics of wavelet transform to estimate the image noise standard deviation, Donoho [6] proposed that image noise can be estimated using the equation sigma $=\mathrm{MAD} / 0.6745$ in the wavelet domain. MAD is the mid-value of HH sub-band wavelet coefficients. But the estimated noise in this method will be large when the noise is small.

Moreover, Konstantinides et al [9] proposed the method that reconstructing and compressing the image after SVD decomposition, then found the data relationship between them to estimate the image noise. LiuWei $[10,11]$ using the average data of the tail singular values to predict the noise strength, through superposing different known noise on image to estimate the original noise standard deviation. Pyatykh et al. [12] proposed a noise variation estimate algorithm based on PCA. After principal component analysis, the minimum PCA coefficient of the image block is chosen as the noise variance under a certain threshold. Wang [13] put forward a kind of noise estimation method based on image segmentation. It segment the image after smoothing it, and select the appropriate variance as the initial value to estimate the noise, then repeat the above steps to modify the estimate parameters to get a more accurate estimation result.

\section{Related Technologies}

Back-propagation Neural Network. BP neural network is a multilayer feedforward network trained by the error back propagation algorithm, and it consists of two processes of the information forward transmission and the error back propagation. Without mapping the relationship of mathematical modeling in advance, BP network can learn and store a lot of nonlinear mapping relationships between input data and output data, so as to handle the complex problems such as classification and prediction. The rule of network learning is using the gradient descent method. BP network is using the error back propagation to adjust the neuron's weights and threshold constantly, to get the minimum error between the network output and the target.

The topological structure of BP neural network includes the input layer, the hidden layer and the output layer. Neurons of Input layer accept a lot of nonlinear data as the input information of the whole network, and transfer them to the middle layer neurons.

The information is input from the input layer, it pass through the hidden layer to output layer, completing a study of the forward propagation process. When the error between actual output and desired output is bigger, the network turn into the error back propagation stage, by this time the error propagate step by step through the output layer to hidden layer and input layer, it modify the weights of each neuron by gradient descent method. The neural network training process is not only the process of information forward transmission and error back propagation, but also the process of adjusting the weights of each layer continually. This process has been performed until the error of the network output is reduced to an acceptable degree or a preset number of iteration.

Singular Value Decomposition of Image. Singular value decomposition (SVD) is an important matrix decomposition in linear algebra, and the decomposed singular value and characteristic vector represent some important features of the data. It has very important applications in signal processing, statistics and other fields.

Let A be zero mean $m \times n$ Gauss white noise matrix with the rank $r$ and the standard deviation $\sigma$, then its SVD decomposition can be expressed as: 


$$
\begin{aligned}
& \mathrm{A}=\mathrm{U} \times \Lambda \times \mathrm{V}^{T} \\
& \sigma^{2}=\sum_{i=1}^{r} \Lambda_{n}^{2}(i)
\end{aligned}
$$

Where $U$ is an $\mathrm{m} \times \mathrm{m}$ unitary matrix, $\Lambda$ is an $m \times n$ diagonal matrix with non-negative real numbers on the diagonal, and the $n \times n$ unitary matrix VT denotes the conjugate transpose of the $n \times n$ unitary matrix $V$. The diagonal entries $\Lambda i$ of $\Lambda$ are known as the singular values of A.

The experiment found that image singular values are influenced by the structure information and the noise. Structure information affect the front large singular values, and noise affect the smaller singular values. Fig.1 shows singular values curve of images under different noise. It can be seen that the distribution of singular value has certain difference in different images, and in the same image, noise intensity mainly influence the end of the singular values. When the noise intensity in image is larger, the tail data of singular values is also greater. Therefore, we can use the singular values of image and neural network to realize the estimation of the noise standard deviation.
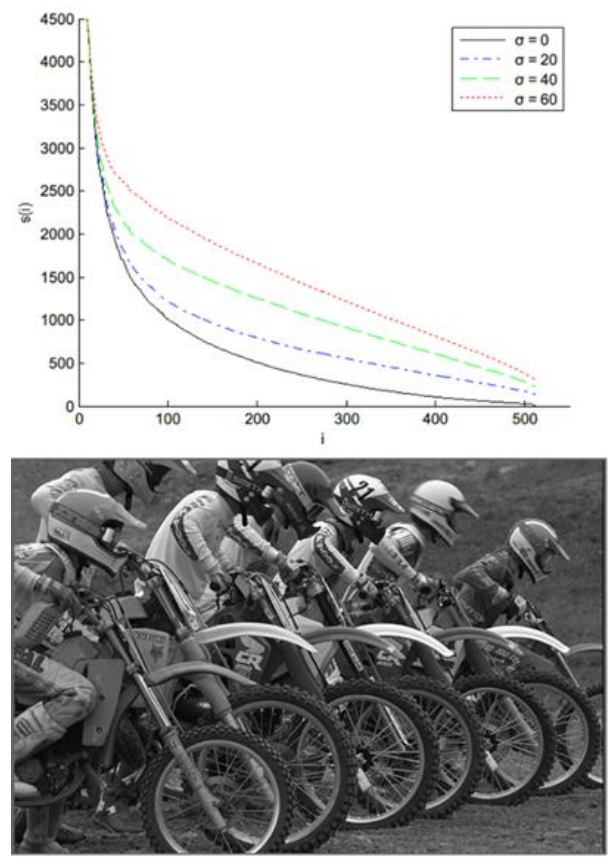

(a)
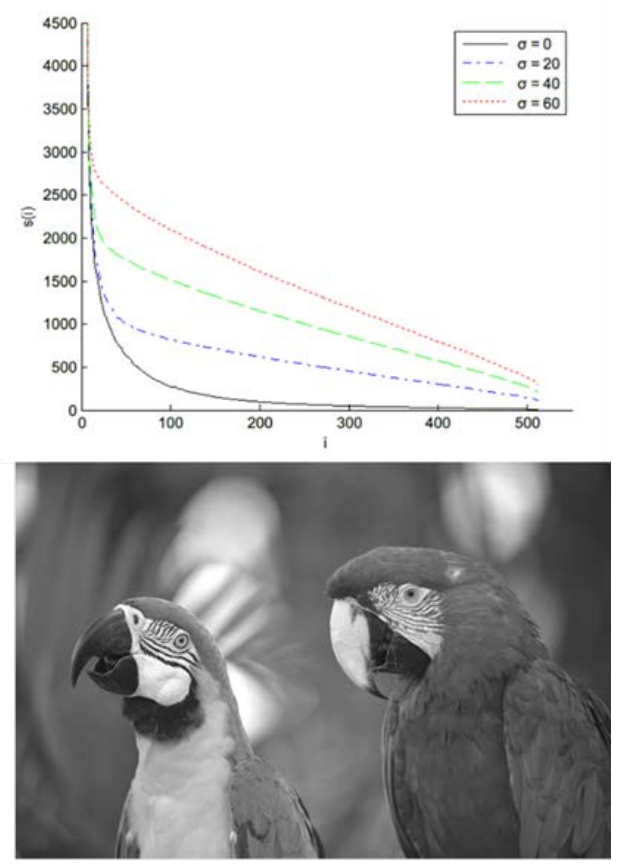

(b)

Fig. 1 Singular values on different noise levels

\section{Image Noise Estimation Based On Neural Network}

We propose a noise estimation algorithm based on neural network and SVD decomposition framework. The framework of proposed algorithm is shown in Fig. 2. In the training stage, we add different intensity of noise on a group of noise free image, and then select a certain number of fixed size image blocks which standard deviation are minimum from these noisy images. After that, decomposing those image blocks using SVD decomposition, and take the decomposed singular values as the input vector of the neural network. At the same time, the noise standard deviation is used as the corresponding output to train the neural network. Therefore, the nonlinear corresponding relationships between the singular values and the noise standard deviation can been obtained. In the prediction stage, it is also select some blocks from test image to estimate the noise intensity, and decompose those image blocks to get their singular value features. After that, inputting these features into the trained neural network, the output value is the estimation of the image noise intensity. 


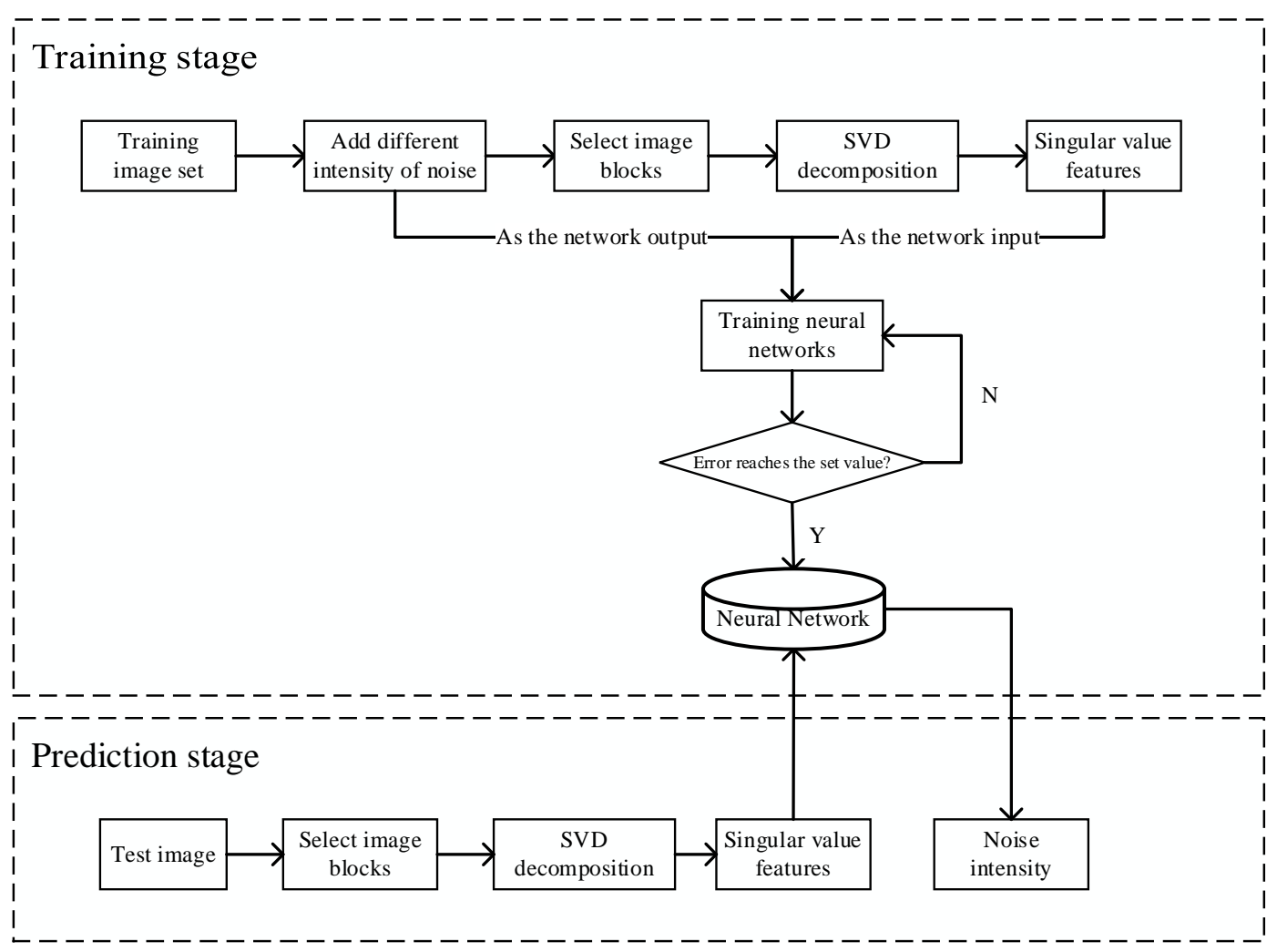

Fig. 2 Noise estimation algorithm framework based on Neural Network

Fig. 3 shows the BP neural network model we used in this paper. The singular value features is input from the input layer, and the dimensions of the input vector determines the number of the neural network input nodes; Hidden layer nodes represent the network model's complexity, and it generally determined by the number of input nodes, the problem complexity and the training samples; Output layer is the predicted standard deviation of noise. Transfer function of hidden layer and output layer neuron is tansig function, as shown in Eq. 5. It maps the input interval $[-\infty,+\infty]$ to the output interval $[-1,1]$, the relationship between input and output is a logistic regression, as shown in Fig. 4 . The training algorithm of the network is using the Levenberg - Marquardt algorithm [14], and its main strategy is to do the linear approximation which use the model function to estimate the parameters of the vector in its neighborhood, ignoring the second order derivative term, so as to convert the problem to linear least squares problems, which has fast convergent rate, and possesses the advantages of gradient method and Newton method.

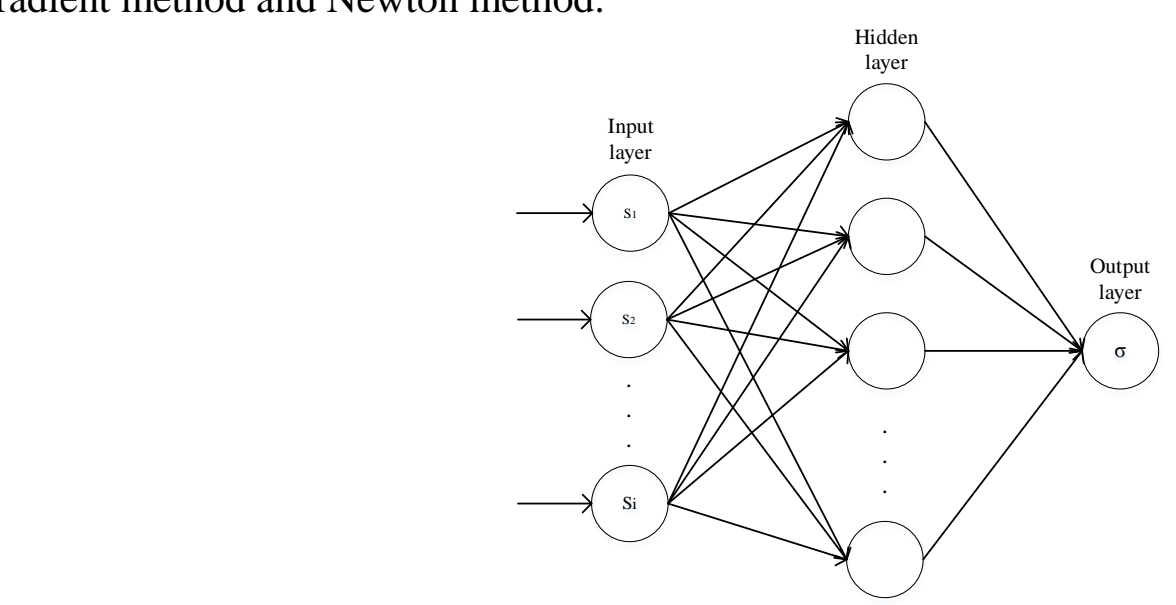

$\mathrm{a}=\tan \operatorname{sig}(\mathrm{n})=\frac{2}{1+e^{(-2 * \mathrm{n})}}-1$

Fig. 3 Neural network model 


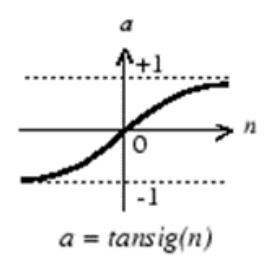

Fig. 4 Tan-Sigmoid transfer function

\section{Experimental Results and Analysis}

To demonstrate the algorithm's performance objectively, we selected 150 noiseless images with various content from Berkeley image segmentation database. And some of them were shown in figure 5. In the experiment, we added the Gaussian White Noise whose standard deviation ranges from 20 to 60 to those images, and put them as training set images. Several fixed-size image blocks with small standard deviation were chosen from each of them, which is used to do SVD decomposition to obtain the singular value features, and then take these decomposed singular values as the input vector of the neural network. At the same time, the noise standard deviation is used as the corresponding output to train the neural network. This experiment is based on single hidden layer BP networks.

Noise Estimation Comparison. In order to verify the accuracy and computational efficiency of the proposed algorithm SVD_NN, we compared this algorithm performance with the PCA algorithm [11] and the SVD2 algorithm [12]. The relative error of estimation is chosen as the standard represents the accuracy of algorithms, and the computation efficiency of algorithms is represented by the average CPU processing time. In our algorithm, the number of image blocks is fixed to 20, the block size is $64 \times 64$, and the number of hidden nodes is assigned to 15 based on the experience.

First, we use the trained neural network to estimate the Gaussian Noise Level of a 512x512 image, after repeating 100 times, results are shown in Table 1.

From the Table 1, we notice that PCA algorithm has larger relative error, and the computational complexity is relatively high as well, so the time of the estimation is longer correspondingly, while the SVD2 algorithm is more accurate and faster than PCA. Compared with these two, our SVD_NN algorithm performed much better, which is faster with higher accuracy.

Table 1 Different algorithm estimation results on Gaussian noise

\begin{tabular}{c|ccccccc}
\hline Algorithm & $\sigma=20$ & $\sigma=30$ & $\sigma=40$ & $\sigma=50$ & $\sigma=60$ & Error (\%) & Time(s) \\
\hline PCA & 20.05 & 30.13 & 40.12 & 49.94 & 59.85 & 0.27 & 2.58 \\
SVD2 & 20.03 & 30.06 & 40.17 & 50.12 & 60.09 & 0.23 & 0.51 \\
SVD_NN & 20.03 & 29.96 & 40.06 & 50.04 & 59.95 & 0.12 & 0.21 \\
\hline
\end{tabular}

To validate the performance when it deals with mixed noise, we added test images with gaussian noise and impulse noise. Then estimate the mixed noise with different intensity level by trained neural network, and the results are shown in Table 2.

Table 2 Different algorithm estimation results on Hybrid noise

\begin{tabular}{c|cccccc}
\hline Algorithm & $\sigma=36.04$ & $\sigma=42.58$ & $\sigma=50.14$ & $\sigma=57.98$ & Error (\%) & Time(s) \\
\hline PCA & 22.03 & 31.32 & 41.49 & 51.42 & 23.47 & 2.24 \\
SVD2 & 35.68 & 42.69 & 49.63 & 58.86 & 0.95 & 0.53 \\
SVD_NN & 36.38 & 43.15 & 49.89 & 58.32 & 0.84 & 0.23 \\
\hline
\end{tabular}

We can see that the PCA algorithm can only estimate the Gauss white noise from the mixed noise. This might because the PCA algorithm assumes that the influence of noise on image is much less than the structural features, and the noise level is represented by the smaller eigenvalue after the blocked PCA transform. But if there are noise impulses with higher amplitude, this assumption is no longer trustable, which leads to serious estimation error. SVD2 algorithm can relatively and accurately estimate the mixed noise, but our algorithm is much better at both accuracy and speed. 


\section{Conclusion}

The singular values of image allows us to analyze the image content from different angles, and separates the main structural information of image from random noise to a certain extent. Combined with strong ability of nonlinear regression of the BP neural network, this paper puts forward a kind of accurate and efficient image noise intensity estimation algorithm. By comparing with currently two efficient noise estimation algorithm, we can draw conclusions that: (1) compared with PCA algorithm, this algorithm can adapt to different kinds of noise, not only for images containing gaussian white noise can estimate the noise standard deviation accurately, the mixed noise can also achieved good results; (2) relative to the SVD2 algorithm of linear estimates, our nonlinear estimation algorithm based on neural network can achieve a more accurate result, and it also can be adapted to different size of images; (3) At the same time, this paper's algorithm on run time is faster than the PCA algorithm and SVD2 algorithm. To sum up, this algorithm has more advantages in the practical applications.

\section{References}

[1] Abramov S K, Lukin V V, Vozel B, et al. Segmentation-based method for blind evaluation of noise variance in images [J]. Journal of Applied Remote Sensing, 2008, 2(1):183-198

[2] Uss M, Vozel B, Lukin V, et al. Image Informative Maps for Estimating Noise Standard Deviation and Texture Parameters [J]. Journal on Advances in Signal Processing, 2011, 6854(5):589-597

[3] Shin D, Park R, Yang S, et al. Block-based noise estimation using adaptive Gaussian filtering [J]. Consumer Electronics IEEE Transactions on, 2005, 51(1):218-226

[4] WANG Z, ZHANG L. An adaptive fast non-local image denoising algorithm [J]. Joumal of Image and Graphics, 2009, 14(4): 669-675

[5] Yang S M, Tai S C. Fast and reliable image-noise estimation using a hybrid approach [J]. Journal of Electronic Imaging, 2010, 19(3): 033007-033007-15

[6] Donoho D L, Johnstone I M. Ideal spatial adaptation via wavelet shrinkage [C] //Biometrika. 1994

[7] De Stefano A, R. White P, B. Collis W. Training methods for image noise level estimation on wavelet components [J]. Eurasip Journal on Applied Signal Processing, 2004, 2004(16):2400-2407

[8] Hashemi M, Beheshti S. Adaptive Noise Variance Estimation in BayesShrink [J]. IEEE Signal Processing Letters, 2010, 17(1):12-15

[9] Konstantinides K, Natarajan B, Yovanof G S. Noise estimation and filtering using block-based singular value decomposition [J]. IEEE transactions on image processing: a publication of the IEEE Signal Processing Society, 1996, 6(3): 479-483

[10] Liu W. Gaussian Noise Level Estimation in SVD Domain for Images. Journal of Image and Graphics, 2012, 17(8): 923-933

[11] Liu W, Lin W. Additive White Gaussian Noise Level Estimation in SVD Domain for Images [J]. IEEE Trans Image Process, 2012, 22(3):872-883

[12] Pyatykh S, Hesser J, Zheng L. Image noise level estimation by principal component analysis [J]. Image Processing, IEEE Transactions on, 2013, 22(2): 687-699

[13] Wang Z. Two-Step Noise Variation Estimation Based on Image Segmentation [C] //Proceedings of the 2013 International Conference on Computer Sciences and Applications. IEEE Computer Society, 2013: 631-634

[14] Hagan M T, Menhaj M B. Training feedforward networks with the Marquardt algorithm [J]. Neural Networks, IEEE Transactions on, 1994, 5(6): 989-993 